\title{
LESÕES MUSCULOESQUELÉTICAS EM POLICIAIS MILITARES
}

\author{
MUSCULOSKELETAL INJURIES IN MILITARY POLICE \\ LESIONES MUSCULOESQUELÉTICAS EN POLICÍAS MILITARES
}

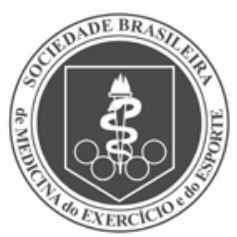

Artigo Original

\author{
Diego Apolinário Calasans \\ (Fisioterapeuta) ${ }^{1}$ \\ Gabriela Borin (Fisioterapeuta) ${ }^{2}$ \\ Gabriel Theodoro Peixoto \\ (Fisioterapeuta) $)^{2}$ \\ 1. Centro de Reabilitação da Polícia \\ Militar - São Paulo, SP, Brasil. \\ 2. Centro de Traumatologia do \\ Esporte/Universidade Federal de \\ São Paulo - São Paulo, SP, Brasil.

\section{Correspondência:} \\ Rua Flores de Goiás, 30 A, Jd. \\ Paulistano - 02814-050 - São Paulo, \\ SP, Brasil. \\ dr.diegoa.calasans@gmail.com
}

\begin{abstract}
RESUMO
Introdução: As qualidades físicas precisam ser analisadas e estão associadas como fatores de risco a desenvolver lesões musculoesqueléticas durante o treinamento esportivo militar. Objetivo: Levantar a epidemiologia das lesões musculoesqueléticas ocorridas em tornozelo e pé de policiais militares. Materiais e Métodos: Foram coletados todos os prontuários de policiais militares que sofreram lesões prévias no tornozelo e pé durante o período de setembro de 2005 a agosto de 2011, as informações foram obtidas através da ficha de avaliação fisioterapêutica constatada nos prontuários, posteriormente os dados obtidos foram tabulados e analisados. Resultados: Após a coleta de dados dos prontuários foi observado que houve $29 \%$ de lesões ósseas, 32\% de ligamentares e 35\% de musculares. Conclusão: A entorse de tornozelo demonstra um risco à saúde pública como descrita pela classificação estatística internacional de doenças e problemas relacionados à saúde, sendo no meio militar também descrita como um risco durante a prática esportiva.
\end{abstract}

Palavras-chave: epidemiologia, exercício físico, prontuários.

\section{ABSTRACT}

Introduction: The physical qualities need to be analyzed and are risk factors associated with the development of musculoskeletal injuries during military sports training. Objective: Epidemiological studies of musculoskeletal injuries occurred in the ankle and foot of military police officers. Methods: We collected all the medical records of military police officers who have suffered previous injuries in the ankle and foot during the period September 2005 to August 2011. The information was obtained through physical therapy evaluation form found in the records and subsequently the data were tabulated and analyzed. Results: After collecting the data from the medical records, it was found that there 29\% bone injuries, 32\% ligament injuries and 35\% muscle injuries. Conclusion: A sprained ankle demonstrates a risk to public health is described by the international statistical classification of diseases and related health problems, which is also in the military environment, described as risk during sports practice.

Keywords: epidemiology, exercise, medical records.

\section{RESUMEN}

Introducción: Las cualidades físicas precisan ser analizadas y se vinculan como factores de riesgos para desarrollar lesiones musculoesqueléticas durante el entrenamiento deportivo-militar. Objetivo: Recolectar datos sobre epidemiología de las lesiones musculoesqueléticas ocurridas en tobillos y pies de policías militares. Materiales y Métodos: Se analizaron todas las fichas médicas de policías militares que sufrieron lesiones previas en tobillos y pies durante el período de septiembre de 2005 a agosto de 2011; las informaciones fueron obtenidas mediante los formularios de evaluación fisioterapéutica que se encontraban en las fichas médicas; posteriormente, los datos obtenidos fueron tabulados y a nalizados. Resultados: Después de la recolección de datos delas fichas médicas se observó 29\% de lesiones óseas, 32\% de ligamentarias y 35\% de musculares. Conclusión: La torcedura de tobillo demuestra ser un riesgo para la salud pública como se describe en la clasificación estadística internacional de enfermedades y problemas relacionados con la salud, siendo en el medio militar señalada también como un riesgo durante la práctica deportiva.

Palabras clave: epidemiología, ejercicios físicos, fichas médicas.

\section{INTRODUÇÃO}

Os jogos mundiais militares nasceram da necessidade de aproximação das instituições militares por meio de eventos poliesportivos com o princípio do Conselho Internacional do Desporto Militar e no espírito olímpico sem considerações políticas. O surgimento dos jogos teve início na comemoração do $50^{\circ}$ aniversário do fim da Segunda Guerra Mundial com a ratificação da Organização das Nações Unidas ${ }^{\text {. }}$

A necessidade de proporcionar segurança e resultados reflete nos militares as consequências do excesso de treinamentos e competições, levando a um aumento do número de lesões do aparelho locomotor. As causas dessas lesões podem ser atribuídas à ausência de medidas pre- ventivas, exaustão na jornada de trabalho e características que variam em função das particularidades como: idade, sexo, traumas, pressão inadequada dos calçados, diferenças sociais, culturais e étnicas dos grupos e populações demográficas ${ }^{2-5}$.

Estudos epidemiológicos relatam que as qualidades físicas precisam ser analisadas e estão associadas como fatores de risco a desenvolver lesões musculoesqueléticas durante o treinamento esportivo militar ${ }^{4}$. Segundo Wen, os fatores de riscos podem ser divididos em extrínsecos ou intrínsecos ${ }^{6}$.

Os fatores extrínsecos são aqueles direta ou indiretamente ligados à preparação ou prática da atividade física: erros de planejamento e execução do treinamento (intensidade, frequência, duração, descanso, periodização, 
prática de aquecimento e alongamento), tipo de superfície de treino (areia, asfalto, grama, concreto), tipo de percurso (declive, tortuoso, plano, irregular), tipo de calçado, alimentação (consumo energético total, álcool ou drogas) e prática concomitante de outras modalidades esportivas ${ }^{6}$.

Já os fatores intrínsecos que supostamente predispõem às lesões são aqueles inerentes ao organismo e incluem: alterações biomecânicas e anatômicas (pés, tornozelo, calcâneo, tíbia, joelhos, assimetrias de comprimento dos membros inferiores), flexibilidade, histórico de lesões, características antropométricas (peso, altura, IMC), densidade óssea e composição corpórea e condicionamento cardiovascular variando com a atividade física do atleta6 .

De acordo com Ejnisman et al. ${ }^{7}$, todas as lesões podem ser classificadas como: lesões traumáticas, provenientes de um trauma causado por contato direto ou indireto mais frequente durante a fase de treinamento ou na competição; e lesões atraumáticas, causadas principalmente por sobrecarga nos tecidos. As lesões que ocorrem por sobrecarga acontecem com uma menor frequência, porém são lesões mais graves.

Jones e Knapik estudaram as lesões causadas pela alta intensidade de treinamento físico de atletas e soldados, e sugeriram o levantamento periódico para determinar o número de lesões, caracterizando as causas e os fatores de riscos que desencadeiam as lesões, adequação do tempo de intervenção e intensidade de exercícios para reduzir as lesões, intervenções preventivas efetivas e o monitoramento do tempo de intervenção para manter a sua eficácia2-4,8-11.

Por causa de sua localização anatômica, o tornozelo e o pé são locais de maior acometimento das lesões, submetidos a forças externas que são absorvidas pelas estruturas passivas (tendões, ligamentos, fáscia e ossos) e por estabilizadores dinâmicos, tecidos contráteis (músculos) periarticulares ${ }^{8}$. O tornozelo é uma articulação composta, que consiste em uma sindesmose entre as extremidades distais da tíbia e fíbula em um encaixe diartrodial junto ao tálus ${ }^{12,13}$. De acordo com sua conformação óssea, quando está em posição neutra é uma articulação com grande estabilidade estática, e com a realização de flexão sua estabilidade diminui pelo deslizamento anterior do tálus fazendo com que sua porção posterior fique no encaixe, permitindo a movimentação lateral13; nesta posição ocorrem $60 \%$ das lesões musculoesqueléticas durante toda prática esportiva ${ }^{14,15}$.

A quantificação das lesões mais frequentes e a identificação das características poderão ajudar na elaboração de planos de tratamentos multidisciplinares e de métodos de prevenção, que contribuirão para diminuir a exposição dos policiais militares aos riscos de lesões.

A Polícia Militar possui mais de 190 mil profissionais, os quais, durante sua carreira, devem manter o condicionamento físico. A ocorrência de lesões relacionadas à prática de atividade física é considerada como problema de saúde pública em países desenvolvidos, onde a população militar tem sido objeto de inúmeras investigações, porém, em nosso meio, são raros os estudos referentes a este assunto.

\section{MATERIAS E MÉTODOS}

Neste estudo foram coletados 272 prontuários de policiais militares atendidos no Centro de Reabilitação da Polícia Militar que apresentaram lesões musculoesqueléticas de tornozelo e pé de setembro de 2005 a agosto de 2011. O levantamento dos prontuários foi realizado com autorização do Major PM Daniel Cesar Simões Teixeira, Chefe Interino do Centro de Reabilitação da Polícia Militar, protocolo no 1481, após a aprovação do Comitê de Ética em Pesquisa da Universidade Federal de São Paulo.

Trata-se de um estudo observacional do tipo transversal retrospectivo.

As informações foram coletadas por um único pesquisador e transferidas para formulário de coleta de dados (anexo 1). A identidade e informações dos policiais militares foram preservadas.
Critérios de inclusão: ser policial militar da ativa de ambos os sexos com idade entre 18 e 40 anos, realizar tratamento no Centro de Reabilitação da Polícia Militar e que tenha lesão ortopédica prévia no tornozelo e/ou no pé.

Critérios de exclusão: todos os prontuários que apresentaram ausência maior ou igual a $20 \%$ do total das informações coletadas e prontuários de pacientes portadores de doenças do sistema nervoso central.

\section{Análise estatística}

Os dados foram tabulados e armazenados no programa Microsoft Exce $^{\circledR}{ }^{\circledR} 2010$, sendo calculada a média e a realização de gráficos para a análise, respectivamente, dos prontuários e das principais lesões em policiais militares. Por se tratar de uma pesquisa de prevalência a análise estatística foi descritiva.

\section{RESULTADOS}

Os dados foram coletados de 272 prontuários de pacientes que realizaram tratamento no Centro de Reabilitação da Polícia Militar no período de setembro de 2005 a agosto de 2011. Foi constatado que desses prontuários analisados, 177 estavam completos e 95 incompletos, sendo, por consequente excluídos da atual pesquisa (figura 1).

Na análise dos prontuários constatou-se que 25,4\% da população era do sexo feminino (45) e 74,6\% do sexo masculino (132), com média de idade de 30 anos e que realizavam treinamento militar esportivo regularmente sendo tendo a lesão decorrente da mesma, como demonstra a tabela 1.

Dentro dessa pesquisa pôde-se observar a classificação das lesões decorrentes da estrutura lesionada, obtendo as seguintes lesões: 2,3\% condrais, 1,7\% nervosas, 29\% ósseas, 32\% ligamentares e $35 \%$ musculares.

Todas as lesões ligamentares foram decorrentes da entorse, sendo predominante no sexo masculino com 58\% quando comparado ao feminino (42\%), porém acima dos 32 anos verificou-se maior incidência entre as mulheres (figura 2).

Durante o período estudado, as lesões com maiores prevalências foram entorse de tornozelo, fasciite plantar, fratura bimaleolar e a tendinopatia calcânea. Houve 48 fraturas em todo o complexo pé e tornozelo desses, 56\% foram acometidas durante acidentes de motociclismo, estando à fratura bimaleolar presente em todo o período estudado e sendo a segunda maior acometida $(n=12)$ dentro das fraturas, encontrando-se somente atrás da fratura de maléolo lateral $(n=16)$.

\section{DISCUSSÃO}

Após o levantamento e análise de 177 prontuários de policiais militares (132 homens e 45 mulheres), foi comprovada a maior incidência de entorse, fasciite plantar, fratura de maléolo lateral e tendinopatia calcânea entre os policiais militares. Um estudo realizado em 2001 relatou que 83\% dos homens e $87 \%$ das mulheres sofreram lesões em membros inferiores assim

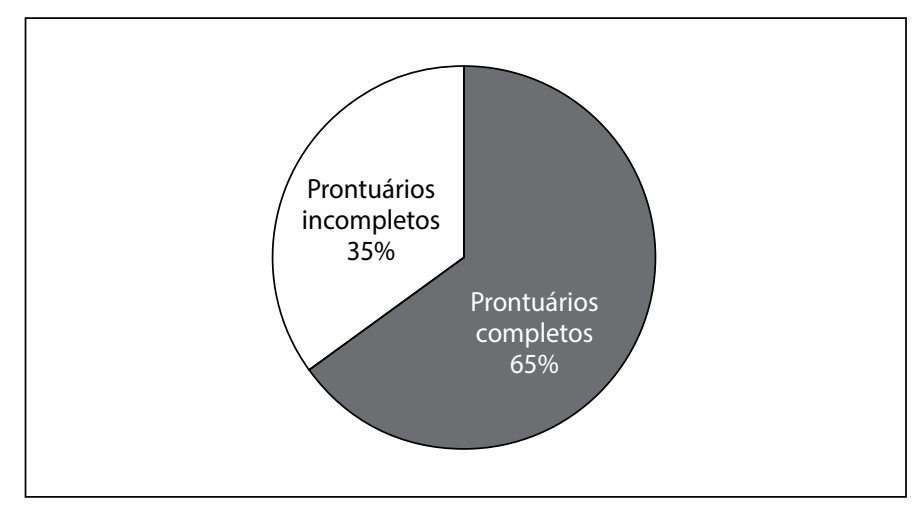

Figura 1. Análise dos prontuários. 
Tabela 1. Dados epidemiológicos de policiais militares.

\begin{tabular}{|c|c|}
\hline Variável & Proporção (\%) \\
\hline Feminino & $45(25,4 \%)$ \\
\hline Masculino & $132(74,6 \%)$ \\
\hline \multicolumn{2}{|c|}{ Média de Idade (Anos) } \\
\hline Feminino & 0 \\
\hline Masculino & 30 \\
\hline \multicolumn{2}{|c|}{ Média de Altura $(\mathrm{cm}) /$ Peso $(\mathrm{kg}) / \mathrm{IMC}$} \\
\hline Feminino & $(165)-(65)-(23,9)$ \\
\hline Masculino & $(176)-(80)-(25,9)$ \\
\hline \multicolumn{2}{|c|}{ Patologias } \\
\hline Distrofia Simpática Reflexa & $1(0,56 \%)$ \\
\hline Entorse & $58(32,7 \%)$ \\
\hline Fascíite & $23(13 \%)$ \\
\hline Fratura Bimaleolar & $12(6,7 \%)$ \\
\hline Fratura Calcânea & $2(1,12 \%)$ \\
\hline Fratura Cuneiforme & $1(0,56 \%)$ \\
\hline Fratura Hálux & $1(0,56 \%)$ \\
\hline Fratura II, III, IV, V Metatarsos & $4(2,25 \%)$ \\
\hline Fratura Luxação de Lisfranc & $1(0,56 \%)$ \\
\hline Fratura Maléolo Lateral & $16(9,03 \%)$ \\
\hline Fratura Maléolo Medial & $7(3,95 \%)$ \\
\hline Fratura Navicular & $2(1,12 \%)$ \\
\hline Fratura Tálus & $3(1,7 \%)$ \\
\hline Lesão Retináculo & $1(0,56 \%)$ \\
\hline Lesão de Nervo Fibular Superficia & $1(0,56 \%)$ \\
\hline Luxação de Tendão Fibular & $1(0,56 \%)$ \\
\hline Metatarsalgia & $3(1,7 \%)$ \\
\hline Neuroma Plantar & $1(0,56 \%)$ \\
\hline Tendinite Calcânea & $16(9,03 \%)$ \\
\hline Tendinite Fibulares & $7(3,95 \%)$ \\
\hline Tendinite Tibial Anterior & $3(1,7 \%)$ \\
\hline Tendinite Tibial Posterior & $3(1,7 \%)$ \\
\hline Tenorrafia Calcânea & $1(0,56 \%)$ \\
\hline
\end{tabular}

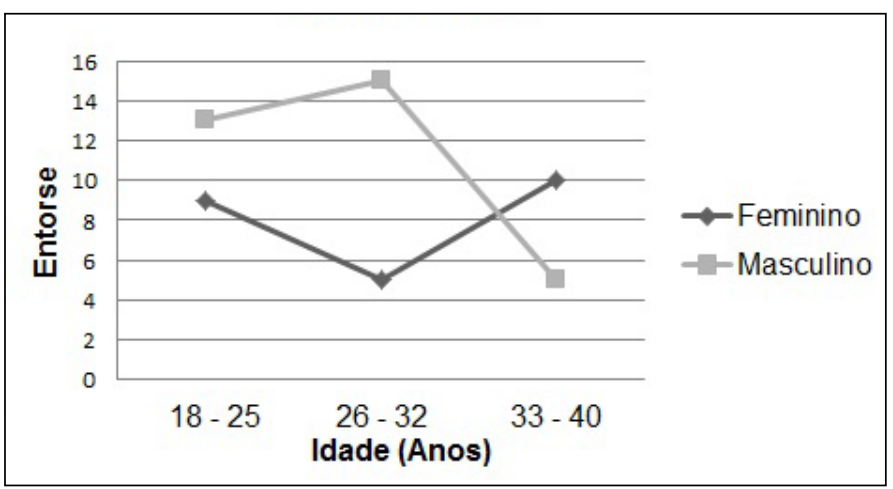

Figura 2. Análise do entorse conforme a idade e o sexo.

que iniciaram a instrução militar e o aprendizado da marcha militar 9,14,15.

Estudos direcionam a atenção para amenizar efeitos deletérios das forças de reação do pé com o solo durante a marcha militar, visto que o passo ordinário é definido como "passo com aproximadamente $75 \mathrm{~cm}$ de extensão, calculado de um calcanhar a outro e numa cadência de 116 passos por minuto"16. A cadência da marcha normal é de 90 passos por minuto ${ }^{11}$, conjecturando que a força exercida pelo impacto do pé durante a marcha pode ser responsável por lesões do aparelho locomotor em recrutas no início do treinamento militar ${ }^{17-20}$.
As lesões ligamentares do tornozelo são as mais comuns que ocorrem, independentemente da função, esporte ou exposição. Um fato apoiado na literatura ${ }^{17}$ constatado em nosso estudo é que as entorses de tornozelo representam 32,7\% de todas as lesões relatadas, sendo de maior prevalência entre o sexo masculino ao feminino, 34 e 24 lesões, respectivamente. Jones et al. observaram que entorses de tornozelo foram as lesões mais prevalentes relacionadas ao treinamento físico entre homens no exército dos EUA ${ }^{2-4}$. Waterman et al. relatam que as taxas de incidência de entorse ligamentares do tornozelo são influenciadas pelo sexo, altura, peso, IMC e condicionamento físico, demonstrado em nossa pesquisa que homens com aumento IMC ocasionado pela maior altura e aumento de peso e exposições esportivas tiveram maior incidência de sofrer entorse de tornozelo ${ }^{17}$.

Considerando o ênfase do treinamento físico que as instituições militares e a alta incidência de lesões relacionadas ao treinamento, em 2009, uma pesquisa descreveu que, aproximadamente, 300 militares foram acompanhados durante as 12 semanas iniciais de treinamento correndo e marchando. A incidência de lesões em membros inferiores por sobrecarga foi de 45,9\%, o padrão de lesões foi determinado em relação ao volume semanal e intensidade de treinamento físico ${ }^{14,15,17}$. Os agravos causados por excesso de treinamento estão presentes em $78 \%$ dos diagnósticos apresentados por Almeida et al. ${ }^{19}$.

Em um estudo de Jones et al., lesões como as tendinopatias e fraturas por estresse foram apontadas como as mais comuns entre os militares. Em nossa análise foi observado que houve 29 casos de tendinopatias; dessas, 55\% foram de calcâneo. Mahieu et al., observaram 69 cadetes masculinos que realizaram o treinamento básico, 14,5\% tiveram tendinopatia calcânea ${ }^{20}$.

Vários autores ${ }^{21-25}$ descrevem as fraturas por estresse como as lesões mais comuns nos militares, encontrando relatos desde 1900; no entanto, a fratura prevalente no presente estudo foi ocasionada por acidente motociclístico. Embora o objetivo desta atual pesquisa seja levantar a epidemiologia das lesões em militares durante a atividade física, vale ressaltar que houve 30 lesões envolvendo motociclismo sendo 28 acometendo o tornozelo dessas 89\% foram fraturas e duas lesões no pé sendo fraturas. Debieux et al. ${ }^{26}$, analisaram prontuários médicos de 2001 a 2002, relatando 96 lesões no tornozelo sendo 12,7\% fraturas e 73 nos pés com 16\% fraturas em motociclistas atendidos no Hospital São Paulo (Brasil).

As limitações do estudo foram a quantidade de prontuários incompletos (95) e as lesões encontradas em motociclista da polícia militar. Por ser uma população militar que apresentava maior prevalência do sexo masculino, o que implica nos resultados da pesquisa, torna-se necessária a realização de novos estudos com grupos mais heterogêneos analisando a incidência de lesões no meio militar e nos esportes individuais.

\section{CONCLUSÃO}

A epidemiologia das lesões ocorridas no complexo tornozelo e pé de policiais militares encontrada neste estudo é similar ao âmbito mundial dos militares citado pela literatura científica durante o treinamento físico. A entorse de tornozelo demonstra um risco à saúde pública, sendo descrita pela classificação estatística internacional de doenças e problemas relacionados à saúde, sendo no meio militar também descrita como um risco durante a prática esportiva.

Foi realizada pesquisa nas principais bases de dados, nas quais não houve qualquer relato de pesquisa analisando os dados epidemiológicos de militares relacionados com os jogos mundiais militares.

Todos os autores declararam não haver qualquer potencial conflito de interesses referente a este artigo. 


\section{REFERÊNCIAS}

1. Taanila H, Parkkari J, Suni J, Mattila VM, Ohrankammen O, Vuorinen P, et al. A etiology and risk factors of musculoskeletal disorders in physically active conscripts: a follow-up study in the Finnish Defense Forces. BMC Musculoskelet Disord 2010;11:146.

2. Jones BH, Cowan DN, Tomlinson JP, Robinson JR, Polly DW, Frykman PN. Epidemiology of injuries associated with physical training among young men in the army. Med Sci Sports Exerc 1993;25:197-203.

3. Jones BH, Bovee MW, Harris JM 3rd, Cowan DN. Intrinsic risk factors for exercise-related injuries among male and female army trainees. Am J Sports Med 1993;21:705-10.

4. Jones HB, Thacker SB, Gilchrist J, Sosin DM, Kimsey Junior CD. Prevention of lower extremity stress fractures in athletes and soldiers: a systematic review. Epidemiol Rev 2004;24:228-47.

5. Knapik JJ. The Army Physical (APFT): a review of the literature. Mil Med 1989;154:326-9.

6. Wen DY. Risk factors for overuse injuries in runners. Curr Sports Med Rep 2007;6:307-13.

7. Ejnisman B, Andreoli CV, Carrera EF, Abdalla RJ, Cohen M. Lesões musculoesqueléticas no ombro do atleta: mecanismo de lesão, diagnóstico e retorno à prática esportiva. Rev Bras Ortop 2001;36:389-93.

8. Knapik JJ, Sharp MA, Darakjy S, Jones SB, Hauret KG, Jones BH. Temporal changes in the physical fitness of US army recruits. Sports Med 2006;36:613-34

9. Knapik JJ, Jones BH, Reynolds K, Ang P. Physical fitness, age, and injury incidence in infantary soldiers. J Occup Med 1993;35:598-603.

10. Knapik JJ, Darakiy S, Hauret KG, Canada S, Scott S, RiegerW, et al. Increasing the physical fitness of low-fit recruits before basic combat training: an evaluation of fitness, injuries, and training outcomes. Mil Med 2006;171:45-54

11. Knapik JJ, Jones BH, Vogel JA, Banderet LE, Bahrke MS, O'Connor JS. Influence of age and body mass index on measures of physical fitness in U.S. Army Soldiers. Journal of Aging and Physical Activity. 1996;4:234-50.

12. Nery CAS. Tornozelo e pé: anatomias funcionais, biomecânicas e semiologia. Em: Cohen M, Abdalla RJ. Lesões nos esportes - Diagnóstico, prevenção e tratamento. Rio de Janeiro: Revinter; 2003. p. 153-72.

13. Cailleit R. Dor no pé e tornozelo 3a ed. São Paulo: Artmed; 2005. p. 19-59.

14. Parkkari J, Taanila H, Suni J, Mattila VM, Ohrankammen O, Vuorinen P, et al. Neuromuscular Training with injury prevention counseling to decrease the risk of acute musculoskeletal injury in young men during military service: A population - based, randomized study. BMC Musculoskelet Disord 2011;9:35.

15. Vender TC, Abt JP, Crawford K, Lovalekar M, Nagai T, Deluzio JB, et al. Warrior model for human performance and injury prevention: Eagle Tactical Athlete Program (ETAP) Part I. J Med Spec Oper 2010;10:2-21.

16. Brasil. Ministério da Defesa. MANUAL C 20 - 20: Treinamento Físico Militar. $3^{\text {a }}$ ed. Rio de Janeiro: Exército; 2002

17. Waterman BR, Belmont PJ Jr, Cameron KL, Deberardino TM, Owens BD. Epidemiology of ankle sprain at the United States Military Academy. Am J Sports Med 2010;38:797-803.

18. Guisande TP, Mochizuki L. Forças de impacto e marcha militar: estudo descritivo. R Educ Tecn Ap Aeron 2009;1:117-23.

19. Almeida SA, Williams KM, Shaffer RA, Brodine SK. Epidemiological patterns of musculoskeletal injuries and physical training. Med Sci Sports Exerc 1999;31:1176-82.

20. Mahieu NN, Witvrouw E, Stevens V, Van Tiggelen D, Roget P. Intrinsic risk factors for the development of Achilles tendon overuse injury: a prospective study. Sports Med 2005;34:226-35

21. Cowan DN, Jones BH, Robinson JR. Foot morphologic characteristics and risk of exercise-related injury. Arch Fam Med 1993;2:773-7.

22. Cowan DN, Jones BH, Frykman PN, Polly DW Jr, Harman EA, Rosenstein RM, et al. Lower limb morphologic and risk of overuse injury among male infantry trainees. Med Sci Sports Exerc 1996;28:945-52.

23. Harting $D E$, Henderson JM. Increasing hamstring flexibility decreases lower extremity overuse injuries in military basic trainees. Am J Sports Med 1999;27:173-6.

24. Kaufaman KR, Brodine S, Shaffer R. Military training-related injuries surveillance, research, and prevention. Am J Prev Med 2000;18:54-63.

25. Milgrom C, Giladi M, Kashtan H, Simkin A, Chisin R, Margulies J, et al. A prospective study of the effect of a shock-absorbing orthotic device on the incidence of stress fractures in military recruits. Foot Ankle 1985;6:101-4

26. Debieux P, Cherman C, Mansur NSB, Dobashi E, Fernandes JA. Lesões do aparelho locomotor nos acidentes com motocicleta. Acta Ortop Bras 2010:18:353-6.

Anexo 1. Formulário de coleta de dados.

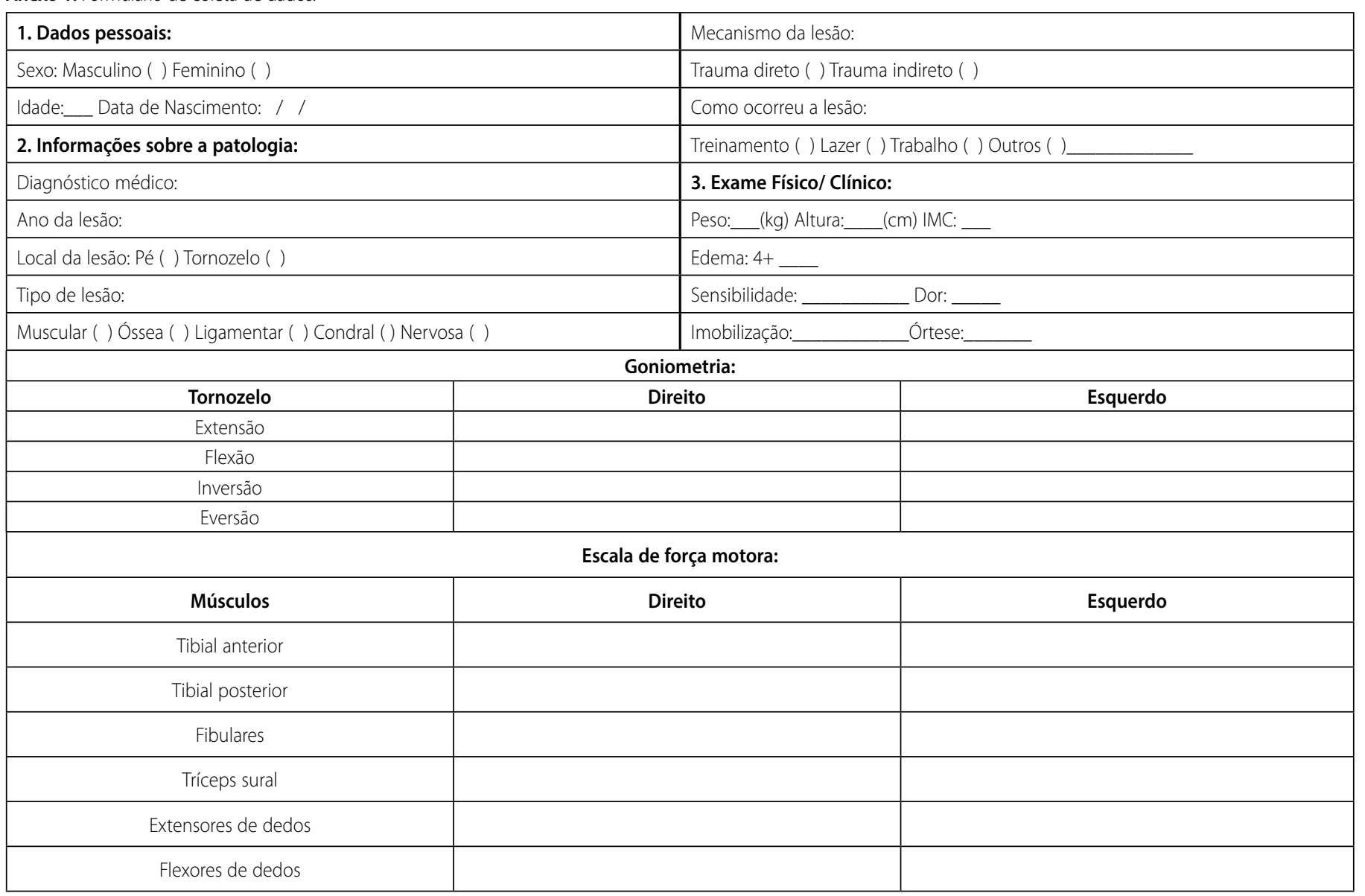

